\title{
Short-Term Load Forecasting Method based on Empirical Wavelet Decomposition and BLSTM Neural Networks
}

This paper was downloaded from TechRxiv (https://www.techrxiv.org).

LICENSE

CC BY-NC-SA 4.0

SUBMISSION DATE / POSTED DATE

$05-09-2021 / 10-09-2021$

\section{CITATION}

Zhang, Xiao-Yu; Kuenzel, Stefanie; Colombo, Nicolo; Watkins, Chris (2021): Short-Term Load Forecasting Method based on Empirical Wavelet Decomposition and BLSTM Neural Networks. TechRxiv. Preprint. https://doi.org/10.36227/techrxiv.16570995.v1

$\mathrm{DOI}$

10.36227/techrxiv.16570995.v1 


\title{
Short-Term Load Forecasting Method based on Empirical Wavelet Decomposition and BLSTM Neural Networks
}

\author{
Xiao-Yu Zhang, Student Member, IEEE, Stefanie Kuenzel, Senior Member, IEEE, Nicolo Colombo, \\ and Chris Watkins
}

\begin{abstract}
Accurate short-term load forecasting is essential to the modern power system and smart grids; the utility can better implement demand-side management and operate the power system stable with a reliable forecasting system. The load demand contains a variety of different load components, and different loads operate with different frequencies. Conventional load forecasting models (linear regression (LR), Auto-Regressive Integrated Moving Average (ARIMA), deep neural network, etc.) ignore frequency domain and can only use time-domain load demand as inputs. To make full use of both time domain and frequency domain features of the load demand, a hybrid component decomposition and deep neural network load forecasting model is proposed in this paper. The proposed model first filters noises via wavelet-based denoising technique, then decomposes the original load demand into several sublayers to show the frequency features while the time domain information is preserved as well. Then bidirectional LSTM model is trained for each sub-layer independently. To better tunning the hyperparameters, a Bayesian hyperparameter optimization algorithm is adopted in this paper. Three case studies are designed to evaluate the performance of the proposed model. From the results, it is found that the proposed model improves RMSE by $66.59 \%$ and $84.06 \%$, comparing to other load forecasting models.

Index Terms - Load forecasting, empirical wavelets transform, BLSTM, data denoising, Bayesian hyperparameter optimization.
\end{abstract}

\section{NOMENCLATURE AND ACRONYMS}

STLF Short-term load forecasting

FFT Fast Fourier transform

WT Wavelets transform

RNN Recurrent neural network

DWT Discrete wavelet transform

IDWT Inverse discrete wavelet transform

EWT Empirical wavelet transform

EMD Empirical mode decomposition

VMD Variational mode decomposition

ML Machine learning

DL Deep learning

LSTM Long-short term memory

BLSTM Bidirectional long-short term memory

GRU Gated recurrent unit

CNN Convolutional neural network

BHO Bayesian hyperparameter optimization

IMF Intrinsic mode functions

AM-FM Amplitude modulation-frequency modulation

SVM Support vector machine

TPE Tree parzen estimators

$Y \quad$ Underlying signal

$F \quad$ Denoised signal

$\begin{array}{ll}\epsilon & \text { White Gaussian noise } \\ \sigma & \text { Intensity of the noise } \\ \varphi_{h} & \text { Low-pass filter } \\ \varphi_{g} & \text { High-pass filter } \\ D[k] & \text { Detail coefficients } \\ A[k] & \text { Approximation coefficients } \\ \rho_{T} & \text { Thresholding function } \\ \hat{f} & \text { Fourier spectrum } \\ \omega_{n} & \text { Support boundaries } \\ T_{n} & \text { Transition area width } \\ N & \text { Number of sub-layers } \\ f_{t} & \text { Forgetting gate } \\ i_{t} & \text { Input gate } \\ \widetilde{C_{t}} & \text { Candidate state value of the cell state } \\ C_{t} & \text { Cell state } \\ C_{t-1} & \text { Cell state of the previous step } \\ O_{t} & \text { Output gate } \\ \sigma & \text { Activation function } \\ T_{L} & \text { Look-back steps } \\ T_{F} & \text { Forecasting steps }\end{array}$

\section{INTRODUCTION}

T oad forecasting is vital for the power system, especially for $\mathcal{L}_{\text {real-time energy management. The prediction results will }}$ influence the utility's plans, including deciding the amount of energy to be generated and purchased in the next stage. However, load forecasting is challenging due to the uncertainty and complexity of the load. Conventional load forecasting techniques, such as linear regression (LR), Auto-Regressive Integrated Moving Average (ARIMA) try to extract features at time domain. Since a variety of load components with different frequencies are contained in the load curve, making the load demand highly nonlinear and nonstationary. These characteristics of the original load demand prevent conventional models from making accurate predictions. In addition, artificial intelligence (AI) based load forecasting methods, especially recurrent neural networks (RNN), achieve desirable accuracy in recent years, RNN models have memory units which not only can learn current input features but also information from the past. This characteristic is extremely suitable for forecasting tasks. However, the same as conventional approaches, although RNN can map no-linear features, it still cannot learn frequencydomain information.

To overcome the limitation of traditional forecasting methods, decomposition techniques are adopted to decouple 
original load demand into sub-layers, and then utilize a deep neural network to learn each sub-layer, respectively. Decomposition methods such as Empirical mode decomposition (EMD), variational mode decomposition (VMD) [1, 2], Seasonal and trend decomposition using loess decomposition (STL decomposition) [3], and empirical wavelet transforms (EWT) [4] are adopted to foresting tasks in literature. In the naïve decomposition approach, STL decomposes the original signal into the level component (the average value of the signal), seasonal component (the repeating cycle of the signal), trend component (the general increasing/decreasing trend of the signal), and remainder component (the noise inside the signal). While EMD, VMD, and EWT utilize TimeFrequency representations for the decomposition.

In the time domain, Fast Fourier transform (FFT), However, FFT is not suitable for non-stationary signal since time information would lose in the frequency domain. Wavelets transform (WT) is superior to FFT since it not only obtains information at the frequency domain but also retains time information. WT and EWT are adopted when the time localization of the spectral components is required, such as analyzing the load demand curve. WT and EWT provide a timefrequency representation of the original time-domain signal. Wavelet transform is widely adopted to solve problems such as stock market forecasting, Electrocardiogram (ECG) signal classification, and load forecasting. As for the load forecasting problem, the portion of load components is varying throughout the day. In this case, knowing the periods of specific spectral components occur is especially important for forecasting load demand. A comparison of the original time-varying load demand curve and power spectrums of the load demand obtained via the DWT decomposition technique is presented in Fig.1. From the figure, it is observed that power spectrums contain not only time-domain information but frequency features.
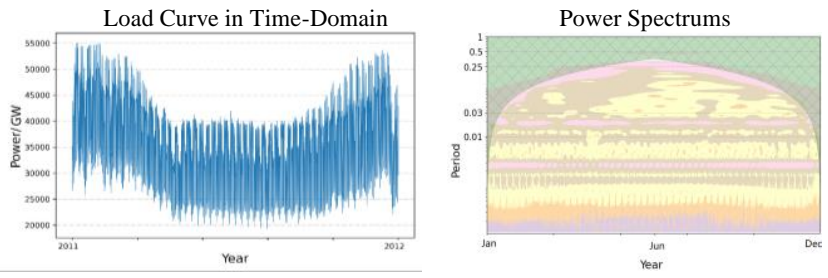

Fig. 1. (a) Load demand curve; (b) Power spectrums of the load demand.

In this paper, a novel short-term load forecasting algorithm based on EWT-BLSTM is proposed. The original load demand data is denoised by wavelets denoising technique to remove noises such as electric spikes. The denoised load demand data is decomposed via the EWT algorithm into sub-compositions. Then BLSTM neural network is adopted to train each sub-layer, respectively. Then each trained BLSTM makes predictions independently, and all predicted sub-layers are reconstructed to present the prediction of load demand. The detailed novelties of this paper are presented as follow:

(1) A novel load forecasting model is proposed. The proposed model combines the EWT component decomposition with a bidirectional LSTM deep neural network to make short- term load forecasting. The performance of the hybrid model can be adjusted by varying the number of sub-layers, lookback window size, and forecasting steps. Moreover, a comparison of the prediction performance among the proposed model with relevant forecasting models (CNNBLSTM/GRU, VMD-LSTM, EMD-LSTM) is implemented. The result shows that the proposed model has better prediction accuracy than other models.

(2) To eliminate the electric spark and other communication noises, a wavelet-based denoising technique is introduced in this paper during the data pre-processing. It is observed that the denoised load demand is smoother than the original curve and noises are eliminated.

(3) A Bayesian Hyperparameter Optimization (BHO) is used for hyperparameter tuning. In bidirectional LSTM, there is an amount of hyperparameter that should be selected (including epoch number, batch size, hidden layer number, etc.). BHO can search the optimal hyperparameters referring to a surrogate probability model, which requires less time and computation ability than traditional optimization methods.

The rest paper is organized as follows: The related work is introduced in section II. The DN-EWT-BLSTM-BHO load forecasting algorithm is demonstrated in Section III. In Section IV, three case studies, which make a comparison among the proposed load forecasting algorithm and other methods, and evaluate the parameters which achieve the best performance, are implemented. The conclusion and final discussion are drawn in the last section.

\section{RELATED WORK}

When Considering the non-stationary and non-linear property of load demand, the combinations of component decomposition with regression algorithms load forecasting models are introduced in literature [2, 5-12]. Component decomposition techniques aim to decompose the original signal into sub-components and extract features at both time and frequency domain. Both EMD, VMD, DWT, and EWT decomposition algorithms are adopted. As an adaptive nonlinear decomposition method, EMD decomposes the original signal into a series of intrinsic mode functions (IMFs) using Hilbert-Huang transform, and each IMF is an amplitude modulation-frequency modulation (AM-FM) signal [7]. EMD can recursively detect upper envelope (local maxima + spline), lower envelope (local minima + spline), and mean envelope (average value of upper and lower envelope), then a sifting process is implemented to obtain an IMF. In contrast to wavelets transform and FFT which have detailed mathematical functions, EMD is an adaptive and fully data-driven method and does not need any predefined function. However, EMD has drawbacks: firstly, EMD lacks the mathematical definition, so it is difficult to understand the decomposition results; secondly, the decomposed signals will diverge at the endpoints and highly sensitive to noise [9]. VMD is an alternative to the EMD algorithm that overcomes the shortages of the EMD method, EWT builds an adaptive wavelet filter bank to replace the EMD. Moreover, the VMD has superior anti-noise ability and separate 
tones of similar frequencies [2, 8, 10]. Another alternative algorithm, the EWT Combines the strength of the wavelet's mathematical definition with the flexibility of EMD.

Once the decomposition components are computed, all components are feed to regression models to make the prediction [13]. The regression models can be divided into two categories, machine learning (ML)-based models and deep learning (DL)-based models. ML methods, which are introduced in $[6,7,9,11,14]$, can be divided into Support Vector Machine (SVM) and its alternative algorithm Least Square - SVM (LS-SVM), Quantile Regression Forest, and SimpleMKL. F. He et al. [11] proposed a hybrid short-term load probability density forecasting method by combing VMD and quantile regression forest, while the Tree-structured of Parzen Estimators (TPE) is used for hyperparameters optimization. Q. Liu et al. and Z. Zhu et al. present a load forecasting method by considering EMD decomposition and SVM model, respectively $[6,7]$. In [6], the SVM model is optimized via the Bat algorithm and the Kalman filtering process, and a fuzzy combination weights theory is used to select similar days for training. In [14], a novel ML algorithm, SimpleMKL, is introduced to make a prediction. Comparing to the conventional SVM, SimpleMKL utilizes L2 norm regularization constraints to solves optimization problems for every single kernel.

DL prediction models, referring to literature, include extreme learning machine (ELM), LSTM, adaptive networkbased fuzzy inference systems (ANFIS) [2, 5, 8, 10]. DL, or deep neural network (DNN), constructed a multi-layer neural network model to map the nonlinear relationship between input features and output. Especially, recurrent neural network (LSTM, GRU) is widely used for solving time series problem for its superior performance in memorizing past information. In [2, 5], S. Kim et al. raised a VMD-LSTM forecasting model. Two-stage decomposition analysis is adopted to process the original load demand curve. The seasonal decomposition divides the original curve into the weekly profiles, then VMD is applied to obtain detailed components. Y. K. Semero proposed an ANFIS-based forecasting model [5]. ANFIS is a neural network based on a fuzzy inference system. So, the model takes advantage of both neural network and fuzzy logic principles. Then a particle swarm optimization (PSO) algorithm is used to optimize the ANFIS model for each IMF component. Finally, A comparison of the VMD-LSTM and conventional methods are presented on a weekday based. Moreover, hybrid ML and DL approaches are introduced in [9]. LS-SVM is adopted to predict low-frequency and intermediate frequency components, while the backpropagation deep neural network is used for predicting high components. And the prediction results from the two models are integrated together to present the final forecasting results.

Although a rich work makes a combination of signal decomposition and ML/DL algorithms, there are still some problems that should be settled and fully investigated. Firstly, electric spikes and other noise would influence the training process and the prediction accuracy, a proper denoising technique should be select to process the original data; secondly, it is proved that EWT has better performance than
DWT, and EMD, choosing EWT as the decomposition model is supposed to have a more promising result. Finally, a hyperparameters tuning algorithm should be proposed to optimize the prediction model.

TABLE I

LOAD FORECASTING MODELS IN THE LITERATURE

\begin{tabular}{|c|c|c|c|}
\hline Reference & $\begin{array}{l}\text { Decomposition } \\
\text { Model }\end{array}$ & $\begin{array}{l}\text { Prediction } \\
\text { model }\end{array}$ & Description \\
\hline [5] & EMD & ANFIS & $\begin{array}{l}\text { PSO algorithm is used } \\
\text { to optimize ANFIS }\end{array}$ \\
\hline$[6,7,12]$ & EMD/ DWT & SVM & $\begin{array}{l}\text { Kalman filtering and } \\
\text { Bat algorithm to } \\
\text { optimize SVM model }\end{array}$ \\
\hline [9] & VMD & $\begin{array}{l}\text { LS- } \\
\text { SVM+DNN }\end{array}$ & $\begin{array}{l}\text { LS-SVM for low and } \\
\text { intermediate frequency } \\
\text { components, DNN for } \\
\text { high-frequency } \\
\text { components }\end{array}$ \\
\hline$[2,8,10]$ & VMD & ELM/ LSTM & $\begin{array}{l}\text { Two-stage } \\
\text { decomposition } \\
\text { (seasonal and VMD) }\end{array}$ \\
\hline [11] & VMD & $\begin{array}{l}\text { Quantile } \\
\text { Regression } \\
\text { Forest }\end{array}$ & $\begin{array}{l}\text { TPE is used for } \\
\text { hyperparameters } \\
\text { optimization }\end{array}$ \\
\hline [14] & EWT & SimpleMKL & $\begin{array}{l}\text { L2 norm regularization } \\
\text { constraints on kernel }\end{array}$ \\
\hline
\end{tabular}

ANFIS: adaptive network-based fuzzy inference systems

LS - SVM: Least Square - Support Vector Machine

ELM: Extreme learning machine

\section{Proposed LoAd ForecAsting Algorithm}

\section{A. Signal Denoising with Wavelets}

The original load data contains a great number of noises, these noises generated from a variety of sources. For example, generated by the electric spikes of electric appliances and intermittent penetration of distributed generators. In addition, measurement devices such as smart meters and SCADA also produce electronic noises. $y_{i}$ represents the original noisy data, while $\epsilon_{i}$ is the white Gaussian noise, and $\sigma$ shows the intensity of the noise. The purpose of the signal denoising is to remove noise and find the best estimation of the underlying signal $y_{i}$ :

$$
y_{i}=f\left(t_{i}\right)+\sigma \epsilon_{i}, i=1, \ldots, n
$$

The denoising process is divided into three steps: signal decomposition, thresholding, and signal reconstruction see Fig. 2.

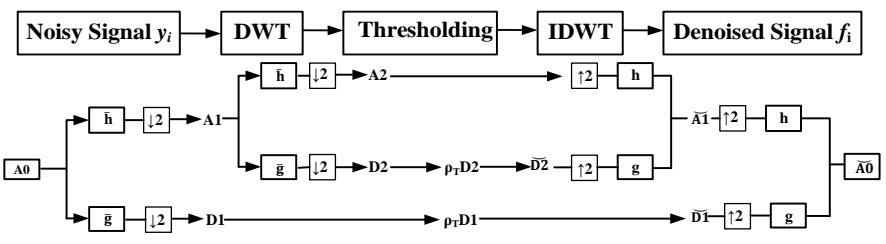

Fig. 2. Block diagram of signal denoising with wavelets

Step 1. Decomposition. The original load demand, which is the noisy signal, is decomposed via Discrete Wavelet Transform 
(DWT), see Fig. 3. The original signal is passed through a series of high-pass and low-pass filters, the detail coefficients are given via high-pass filter and the approximation coefficients are given via low-pass filter. The kth level decomposition functions are:

$$
\begin{aligned}
& h(k)=\sum_{k=-\infty}^{\infty} S[k] \varphi_{h}[2 n-k] \\
& g(k)=\sum_{k=-\infty}^{\infty} S[k] \varphi_{g}[2 n-k]
\end{aligned}
$$

Where $S$ represents the input signal, $\varphi_{h}$ and $\varphi_{g}$ are low-pass and high-pass filters, respectively. And $\mathrm{n}$ is the number of total decomposition levels. After passing each pair of filters, downsampling by two is implemented to compute the lowerlevel detail $D[k]$ and approximation coefficients $A[k]$.

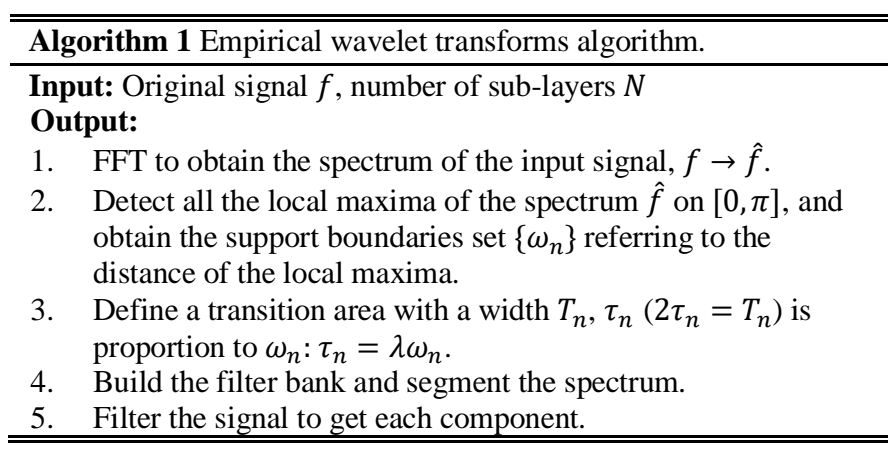

Step 2. Thresholding. Determining the suitable threshold is important for data denoising, a thresholding function $\rho_{T}$ is required. Thresholding can be divided into hard and soft thresholding. As for hard thresholding, the values which exceed the threshold would be set to 0 . And the soft thresholding, the magnitude of coefficients greater than the thresholding is softened. the noise level $\delta_{\text {mad }}$ is first estimated referring to the last level of the detail coefficients by median absolute deviation:

$$
\begin{gathered}
\delta_{\text {mad }}=\frac{\text { median }\left\{\left|c_{0}\right|,\left|c_{1}\right|, \ldots,\left|c_{n}-1\right|\right\}}{0.6745} \\
\text { Threshold, } T=\delta_{\text {mad }} \sqrt{2 \ln (N)}
\end{gathered}
$$

After the threshold determined, the soft thresholding function is applied to reduce the magnitude of the coefficient:

$$
\rho_{T}(x)= \begin{cases}x-T & \text { if } x \geq T \\ x+T & \text { if } x \leq-T \\ 0 & \text { if }|x| \leq T\end{cases}
$$

Step 3. Reconstruction. The coefficients after thresholding are reconstructed again via Inverse Discrete Wavelet Transform (IDWT). Fig.2 makes a comparison between the original load demand curve and the denoised demand curve. From the figure, it is observed that the noises and spikes from the original data are successfully cleared.

\section{B. Empirical Wavelet Transforms (EWT)}

EWT has been widely applied in signal processing and analysis applications, such as seismic data analysis, EEG signals classification, image processing [15]. In power system discipline, EWT has been used for power-quality estimation

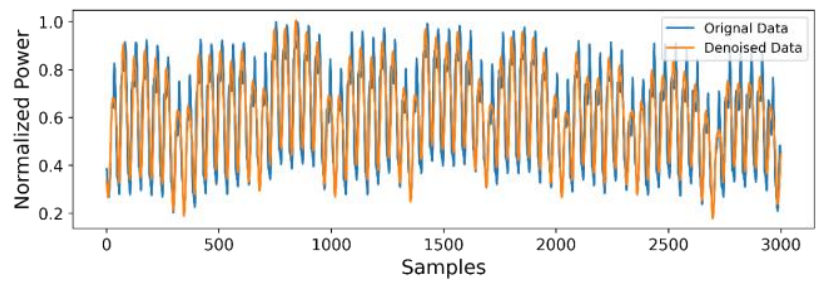

Fig. 3. Comparison between original and denoised load curve (Interval 1 hour)

[16] and short-term load forecasting [14]. Compared with EMD, EWT has a more consistent decomposition and rigorous theoretical foundations $[13,15,17]$. The main idea of EWT is to use spectral modes to replace AM-FM components in EMD. Algorithm 1 shows the pseudocode of the EWT algorithm. From the algorithm, the Fourier spectrum $\hat{f}$ on $[0, \pi]$ is segmented to look for the filter supports. $\mathrm{N}$ segments are obtained, and the length of each segment is determined as $\Lambda_{n}=$ $\left[\omega_{n-1}, \omega_{n}\right]$. Referring to the determined segments, boundaries set $\left\{\omega_{n}\right\}$, as well as transition areas, set $\left\{T_{n}\right\}$ are defined. The scaling function and the wavelet function are defined in (7-8), respectively.

$$
\begin{gathered}
\hat{\phi}_{n}(\omega)= \begin{cases}1 & \text { if }|\omega| \leq(1-\gamma) \omega_{n} \\
\cos \left[\frac{\pi}{2} \beta\left(\frac{1}{2 \gamma \omega_{n}}\left(|\omega|-(1-\gamma) \omega_{n}\right)\right)\right] & \text { if }(1-\gamma) \omega_{n} \leq|\omega| \leq(1+\gamma) \omega_{n}(7) \\
0 & \text { otherwise }\end{cases} \\
\hat{\psi}_{n}(\omega)=\left\{\begin{array}{cc}
1 & \text { if }(1+\gamma) \omega_{n} \leq|\omega| \leq(1-\gamma) \omega_{n+1} \\
e^{-\frac{\omega}{2} \cos }\left[\frac{\pi}{2} \beta\left(\frac{1}{2 \gamma \omega_{n+1}}\left(|\omega|-(1-\gamma) \omega_{n+1}\right)\right)\right] & \text { if }(1-\gamma) \omega_{n+1} \leq|\omega| \leq(1+\gamma) \omega_{n+1}(8) \\
e^{-\frac{\omega}{2} \sin }\left[\frac{\pi}{2} \beta\left(\frac{1}{2 \gamma \omega_{n}}\left(|\omega|-(1-\gamma) \omega_{n}\right)\right)\right] & \text { if }(1-\gamma) \omega_{n} \leq|\omega| \leq(1+\gamma) \omega_{n} \\
0 & \text { otherwise }
\end{array}\right.
\end{gathered}
$$

Where the arbitrary function $\beta$ and the ratio $\gamma$ are defined as:

$$
\begin{aligned}
& \beta(x)= \begin{cases}0 & \text { if } x \leq 0 \\
1 & \text { and } \beta(x)+\beta(1-x)=1 \quad \forall x \in[0,1]\end{cases} \\
& \text { and } \quad \gamma<\min _{n}\left(\frac{\omega_{n+1}-\omega_{n}}{\omega_{n+1}+\omega_{n}}\right) \text {. }
\end{aligned}
$$

Given the scaling function, the detail coefficients and approximation coefficients functions are defined in (9) and

(10), respectively:

$$
\begin{aligned}
& \mathcal{W}_{f}^{\mathcal{E}}(n, t)=\left\langle f, \psi_{n}\right\rangle=\int f(\tau) \overline{\psi_{n}(\tau-t)} d \tau=\left(\hat{f}(\omega) \overline{\hat{\psi}_{n}(\omega)}\right)^{\vee}(9) \\
& \mathcal{W}_{f}^{\mathcal{E}}(0, t)=\left\langle f, \phi_{1}\right\rangle=\int f(\tau) \overline{\phi_{1}(\tau-t)} d \tau=\left(\hat{f}(\omega) \overline{\hat{\phi}_{1}(\omega)}\right)^{\vee}(10)
\end{aligned}
$$

Finally, the decomposed components can be reconstructed via the reconstruction function:

$$
\begin{aligned}
f(t) & =\mathcal{W}_{f}^{\mathcal{E}}(0, t) \star \phi_{1}(t)+\sum_{n=1}^{N} \mathcal{W}_{f}^{\mathcal{E}}(n, t) \star \psi_{n}(t) \\
& =\left(\widehat{\mathcal{W}}_{f}^{\varepsilon}(0, \omega) \hat{\phi}_{1}(\omega)+\sum_{n=1}^{N} \widehat{\mathcal{W}}_{f}^{\varepsilon}(n, \omega) \hat{\psi}_{n}(\omega)\right)^{\vee}
\end{aligned}
$$

\section{Bidirectional Long-short Term Memory (BLSTM)}

Comparing to the conventional deep neural networks which can only capture input information of a single time step, RNN has a recurrent connection to link the previous state with the current input, this structure enables RNN to have good performance in learning sequential information. Proposed by S. Hochreiter and J. Schmidhuber in 1997 [18], BLSTM is a variation of the traditional LSTM algorithm. The LSTM unit 
regulates information relies on a structure called a gate. The gate consists of a sigmoid activation function $\sigma$ and a pointwise multiplication operation. The sigmoid activation function only has " 0 " and " 1 " two values, a value of " 0 " means the gate is closed, and " 1 " means the gate is open and all information can go through the gate. As shown in Fig. 4 (a), there are three gates in the LSTM unit, which are forget gate $f_{t}$, input gate $i_{t}$, and output gate $o_{t}$. With the regulations of the gates, the information of the cell state $C_{t}$ is updated to keep the most important information from the past sequence.

The responsibility of the forget gate is to delete the information from the cell state $C_{t}$. As shown in (12), the forget gate $f_{t}$ takes two inputs, $x_{t}$ and $h_{t-1}$, where $h_{t-1}$ is the hidden state from the previous cell, and $x_{t}$ is the input at the current time step. If the output of $f_{t}$ is closer to " 1 " means to keep, otherwise, the information is forgotten.

$$
f_{t}=\sigma\left(W_{f}\left[x_{t}, h_{t-1}\right]+b_{f}\right)
$$

As for the input gate $i_{t}, x_{t}$ and $h_{t-1}$ is passed into a sigmoid function to decide values to be updated, see (13). Also, $x_{t}$ and $h_{t-1}$ is passed into a tanh function to squish values between [$1,1]$ to creates a new candidate cell state value $\widetilde{C_{t}}$, see (14). Finally, the new cell state $C_{t}$ is determined given $i_{t}$ and $\widetilde{C_{t}}$, shown in (15):

$$
\begin{aligned}
& i_{t}=\sigma\left(W_{i}\left[x_{t}, h_{t-1}\right]+b_{i}\right) \\
& \widetilde{C_{t}}=\tanh \left(W_{c}\left[x_{t}, h_{t-1}\right]+b_{c}\right) \\
& C_{t}=f_{t} \cdot C_{t-1}+i_{t} \cdot \widetilde{C}_{t}
\end{aligned}
$$

Finally, the output of the cell and the hidden state is determined by the output gate $o_{t}$ :

$$
\begin{aligned}
& o_{t}=\sigma\left(W_{o}\left[x_{t}, h_{t-1}\right]+b_{o}\right) \\
& h_{t}=\tanh \left(C_{t}\right) \cdot o_{t}
\end{aligned}
$$

Where $W_{f}, W_{i}, W_{c}, W_{o}$ are weight matrices; and $b_{f}, b_{i}$, $b_{c}, b_{o}$ are the bias.

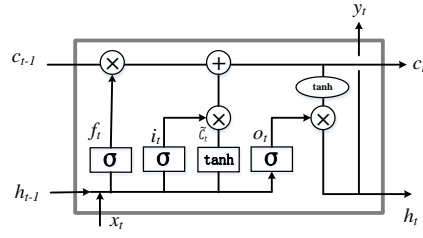

(a)

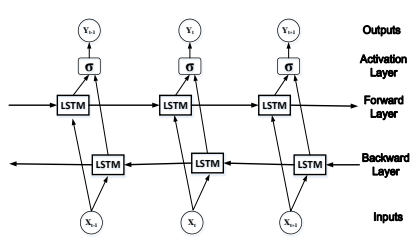

(b)
Fig. 4. (a) LSTM block (b) Bidirectional LSTM

As for bidirectional LSTM (BLSTM), it contains two independent LSTMs which take input in forwarding/ backward direction, respectively, sees Fig. 4 (b). This structure enables the neural network to preserve information from both past and future.

\section{Bayesian Hyperparameter Optimization}

Hyperparameter optimization is essential for training neural networks, the purpose of hyperparameter optimization is to find the hyperparameters that return the best accuracy or performance given a dataset. Conventional hyperparameter optimization approaches, such as grid search and random search, are time-consuming and computation expensive. Comparing to the above methods, a novel Bayesian hyperparameter optimization (BHO) is proposed by Kramer et al. in 2011 [19].
The main methodology of the Bayesian hyperparameter optimization method is to construct a surrogate probability model to select hyperparameters to minizine the original objective function. Providing a sample domain $\mathcal{X}$, The true objective function $f$ which to be optimized is approximated with a surrogate function $\mathcal{M}$. The probability model $\mathcal{M}$ is initialized with a small group of data from $\mathcal{X}$, then an acquisition function $\mathcal{S}$ is adopted to choose the next point to query. A variety of surrogate functions $\mathcal{M}$ are introduced in the literature [20], including Gaussian processes, random forests, tree parzen estimators (TPE), etc. In this paper, hyperparameters include learning rate, drop rate, cell type, etc (shown in Table 2).

TABLE I

HYPERPARAMETER TUNING RANGE.

\begin{tabular}{ll}
\hline \multicolumn{2}{c}{ HYPERPARAMETER TUNING RANGE. } \\
\hline Learning rate & Range \\
Dropout rate & $10^{-5} \sim 10^{-1}$ \\
Cell type & $0.3 \sim 0.7$ \\
Number of hidden layers & GRU, LSTM \\
Batch size & $1 \sim 5$ \\
Optimizer & $32,64,128,256,512,1024$ \\
Loss & Adam, Nadam, RMSprop, Adagrad \\
Activation function & MSE, MAPE, MAE, Huber \\
Epochs & ReLU, Sigmoid, Tanh \\
\hline \hline
\end{tabular}

\section{E. Proposed Load Forecasting Algorithm}

As data denoising technique, EWT decomposition method, BLSTM model, as well as Bayesian hyperparameter optimization are introduced in previous sections, the final step is to combine all these algorithms together to create the hybrid load forecasting model. The block diagram of the proposed model is demonstrated in Fig. 5. Detailed steps are illustrated as follow:

Step 1. Data preparation and data denoising. The original load demand dataset is uploaded to the workspace and visualized by plotting the time series load demand curve. A data cleaning is applied to the original dataset to fill the missing features. Then the wavelet-based denoising algorithm introduced in Section 3.1 is applied to the data to remove the noises. The noised dataset is then split into training/testing datasets, respectively.

Step 2. EWT decomposition. The denoised load demand is decomposed into $N$ sub-layers via the EWT decomposition algorithm introduced in Section 3.2.

Step 3. BLSTM model training. $N$ BLSTM models are constructed and each BLSTM neural network model is trained for one sub-layer. The detailed BLSTM model is demonstrated in Section 3.3.

Step 4. Bayesian hyperparameter optimization. The hyperparameters of each BLSTM model are optimized via BHO to reach the best performance, as illustrated in Section 3.4. Step 5. Load forecasting. The testing set of each sub-layer is used for forecasting to obtain predictions of feature load demand. 
Step 6. EWT reconstruction. Referring to (11), the prediction results for all sub-layers are reconstructed to present the predictions for load demand.

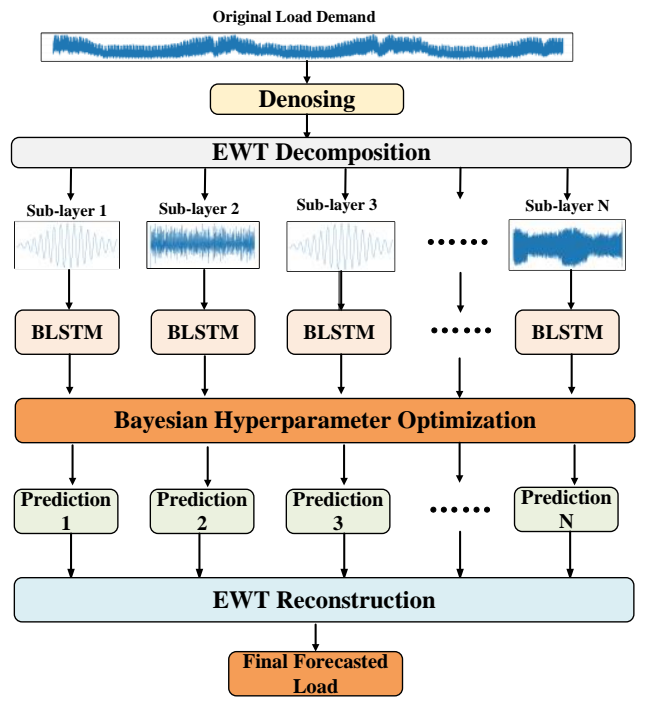

Fig. 5. EWT-BLSTM Load Forecasting Framework.

\section{EXPERIMENTAL SETUP}

\section{A. Data Description and Pre-preparation}

Historic Demand Data collected by National Electricity Transmission System (NETS), UK is adopted as the dataset [21]. The dataset contains the hourly historic demand data, wind generation, solar generation, and interconnector flow in the whole British area, from 2005 to 2020. In this study, the National Demand (ND-Dataset), which is the sum of generation based on National Grid operational generation metering; and England and Wales Demand (EWD-Dataset), which is the sum of generation based on the England and Wales area are selected as the prediction target. The load demand of ND-Dataset and EWD-Dataset is from 2015 to 2016 is used for the simulation. The dataset is split into training/testing sets ( $90 \%$ for training, and $10 \%$ for testing). The original dataset is then denoised via DWT denoising described in Section 3.1, then the denoised dataset is normalized via the max-min normalization, so all data is limited between $[-1,1]$.

\section{B. Open Access Software Platform and Package}

To implement the proposed simulation case study, a variety of open access packages and libraries based on Python 3.7 and TensorFlow 2 is adopted. PyWavelets [22], PyEMD [23], ewtpy [24], vmdpy [24] are used for implementing DWT, EMD, EWT, VMD, respectively. A Bayesian hyperparameter optimization package, Hyperopt [25] is used for hyperparameter tuning.

\section{Performance Metrics}

To assess the performance of the proposed predictor, four performance metrics are adopted, which are Mean Absolute Error (MAE), Mean Absolute Percentage Error (MAPE), Rooted Mean Squared Error (RMSE), and $R^{2}$. Detailed formulas are shown as follow:
(1) Mean Absolute Error (MAE):

$$
M A E=\frac{\sum_{i=1}^{N}\left|y_{i}-\hat{y}_{i}\right|}{N}
$$

(2) Mean Absolute Percentage Error (MAPE):

$$
M A P E=\frac{\sum_{i=1}^{N}\left|\left(y_{i}-\hat{y}_{i}\right) / y_{i}\right|}{N} \times 100 \%
$$

(3) Rooted Mean Squared Error (RMSE):

(4) $R^{2}$ :

$$
R M S E=\sqrt{\frac{\left(\sum_{i=1}^{N}\left[y_{i}-\hat{y}_{i}\right]^{2}\right)}{N}}
$$

$$
R^{2}=1-\frac{S S_{R E S}}{S S_{T O T}}=1-\frac{\sum_{i}\left(y_{i}-\hat{y}_{i}\right)^{2}}{\sum_{i}\left(y_{i}-\bar{y}\right)^{2}}
$$

where $S S_{R E S}$ is sum squared regression error, $S S_{T O T}$ is sum squared total error.

\section{RESULTS AND DISCUSSION}

To evaluate the proposed load forecasting model, two datasets are tested, which are ND-dataset and EWD-dataset, as mentioned above. Three case studies are designed in this section: the influence of $\mathrm{N}$ on the forecasting performance, the influence of look back steps $T_{L}$ and prediction steps $T_{F}$, and finally a comparison with the proposed model and relevant works.

\section{A. Impact of the Number of Sub-layers $N$}

Referring to the EWT decomposition technique introduced in Section 3.2, the original time-varying load demand is decomposed into $N$ sub-layers by EWT, which is named as $S I \sim S N$ in this study. The number of $N$ has a great impact on the final forecasting performance. In this study, the range of $N$ is increasing from 5 to 13, both ND-Dataset and EWD-Dataset are used as this part of the comparison experiment. The performance of the proposed model with different numbers of $N$ are summarized in Tables 2 and 3, and Fig. 6. From these tables, it is observed that the MAE, MAPE, and RMSE are relatively large as $N$ is too small (near 5) or too large (near 13) (See Fig.6). As for ND-Dataset, among all $N$ values, the dominant one is $N=9$, followed by $N=11$, where the RMSE values are $370.312 \mathrm{GW}$ and $380.409 \mathrm{GW}$. As for EWD-Dataset, the proposed model achieves the best performance (smallest value of MAE, MAPE, and RMSE) when $N=12$.

The predictions for decomposed sublayers given the testing set are shown in Fig.6. The load demand is decomposed into $N$ sub-layers by EWT which provides the best performance of selected datasets ( $N=9$ for ND-Dataset and $N=12$ for EWD-Dataset). From the figure, the blue bold line represents the ground truth values, while the red line represents the predicted values. As for high-frequency components sublays(S1 S4), the proposed model has good prediction performance. While the high-frequency components are high fluctuations, and most prediction errors come from the prediction for these components.

$$
\text { TABLE II }
$$

PREDICTION PERFORMANCE OF THE PROPOSED MODEL WITH DIFFERENT SUBLAYER NUMBERS (ND-DATASET).

\begin{tabular}{lllll}
\hline \hline $\mathrm{N}$ & MAE (GW) & MAPE (\%) & RMSE (GW) & $\mathrm{R}^{2}$ \\
\hline
\end{tabular}



NEURAL NETWORKS

\begin{tabular}{lllll}
\hline 5 & 582.978 & 2.011 & 711.649 & 0.983 \\
6 & 392.633 & 1.367 & 555.707 & 0.990 \\
7 & 371.902 & 1.251 & 532.491 & 0.991 \\
8 & 508.232 & 1.648 & 613.607 & 0.987 \\
$\mathbf{9}$ & $\mathbf{2 7 1 . 2 4 3}$ & $\mathbf{0 . 9 1 4}$ & $\mathbf{3 7 0 . 3 1 2}$ & $\mathbf{0 . 9 9 6}$ \\
10 & 484.499 & 1.670 & 578.157 & 0.988 \\
11 & 277.304 & 0.990 & 380.409 & $\mathbf{0 . 9 9 6}$ \\
12 & 794.392 & 2.588 & 873.456 & 0.975 \\
13 & 458.741 & 1.581 & 555.459 & 0.990 \\
\hline \hline
\end{tabular}

TABLE III

PREDICTION PERFORMANCE OF THE PROPOSED MODEL WITH THE DIFFERENT SUB-LAYER NUMBERS (EWD-DATASET).

\begin{tabular}{lllll}
\hline \hline $\mathrm{N}$ & MAE $(\mathrm{GW})$ & MAPE $(\%)$ & RMSE $(\mathrm{GW})$ & $\mathrm{R}^{2}$ \\
\hline 5 & 368.726 & 1.080 & 472.883 & 0.979 \\
6 & 401.092 & 1.213 & 510.023 & 0.978 \\
7 & 239.131 & 0.722 & 313.860 & 0.992 \\
8 & 230.045 & 0.680 & 296.049 & 0.992 \\
9 & 324.022 & 0.958 & 385.466 & 0.987 \\
10 & 301.210 & 0.895 & 371.009 & 0.988 \\
11 & 279.194 & 1.044 & 418.493 & 0.993 \\
$\mathbf{1 2}$ & $\mathbf{1 8 9 . 6 1 4}$ & $\mathbf{0 . 5 5 8}$ & $\mathbf{2 5 6 . 7 0 9}$ & $\mathbf{0 . 9 9 4}$ \\
13 & 229.499 & 0.681 & 297.396 & 0.992 \\
\hline \hline
\end{tabular}

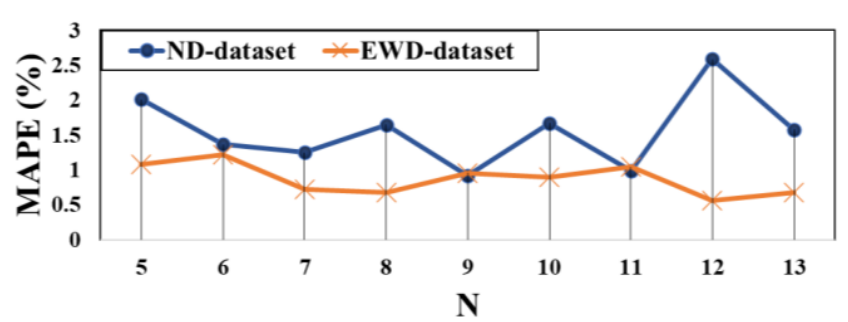

Fig. 6. MAPEs of the proposed model with different sub-layer number.

\section{B. Impact of Look-Back Steps $T_{L}$ and Forecasting Steps $T_{F}$}

In this case study, the look-back steps $T_{L}$ as well as forecasting steps $T_{F}$ are roughly tuned to look for the parameters to achieve the best performance. In the previous study, only one step ahead (one hour) is predicted, while $T_{F}$ means the model will make predictions for several steps further (the interval is one hour for each step). As for the look back steps $T_{L}$, it defines how many previous timesteps are used in order to predict the future load demand.

We vary $\mathrm{T}_{\mathrm{L}}$ and $\mathrm{T}_{\mathrm{F}}$ from 1 to 9 using an interval of 1 , and we applied the load forecasting model using every combination of $\mathrm{T}_{\mathrm{L}}$ and $\mathrm{T}_{\mathrm{F}}$. Fig. 8 provides the $R^{2}$ of the load forecasting model across the whole testing set for different $\mathrm{T}_{\mathrm{L}}$ and $\mathrm{T}_{\mathrm{F}}$ combinations. As for ND-Dataset shown in Fig. 8 (a), the $R^{2}$ is relatedly large as $\mathrm{T}_{\mathrm{L}}$ increases and $\mathrm{T}_{\mathrm{F}}$ decreases since a large look-back step enable the LSTM neural network to learn more information from historical data. Meanwhile, a large forecasting step adds more uncertainty, which reduces the efficiency of the proposed model. From the figure, when $T_{L}$ ranges $2-6$, and $T_{F}$ is 1 , the proposed load forecasting model reaches the best accuracy. As for EWD -Dataset shown in Fig. 8 (b), the proposed model has good prediction ability even given large $\mathrm{T}_{\mathrm{F}}\left(R^{2}>0.95\right)$. Among all presented combinations, the proposed model with $\mathrm{T}_{\mathrm{L}}$ ranges $2-5$, and $\mathrm{T}_{\mathrm{F}}$ is 1 achieves the highest $R^{2}\left(R^{2}=0.99\right)$.

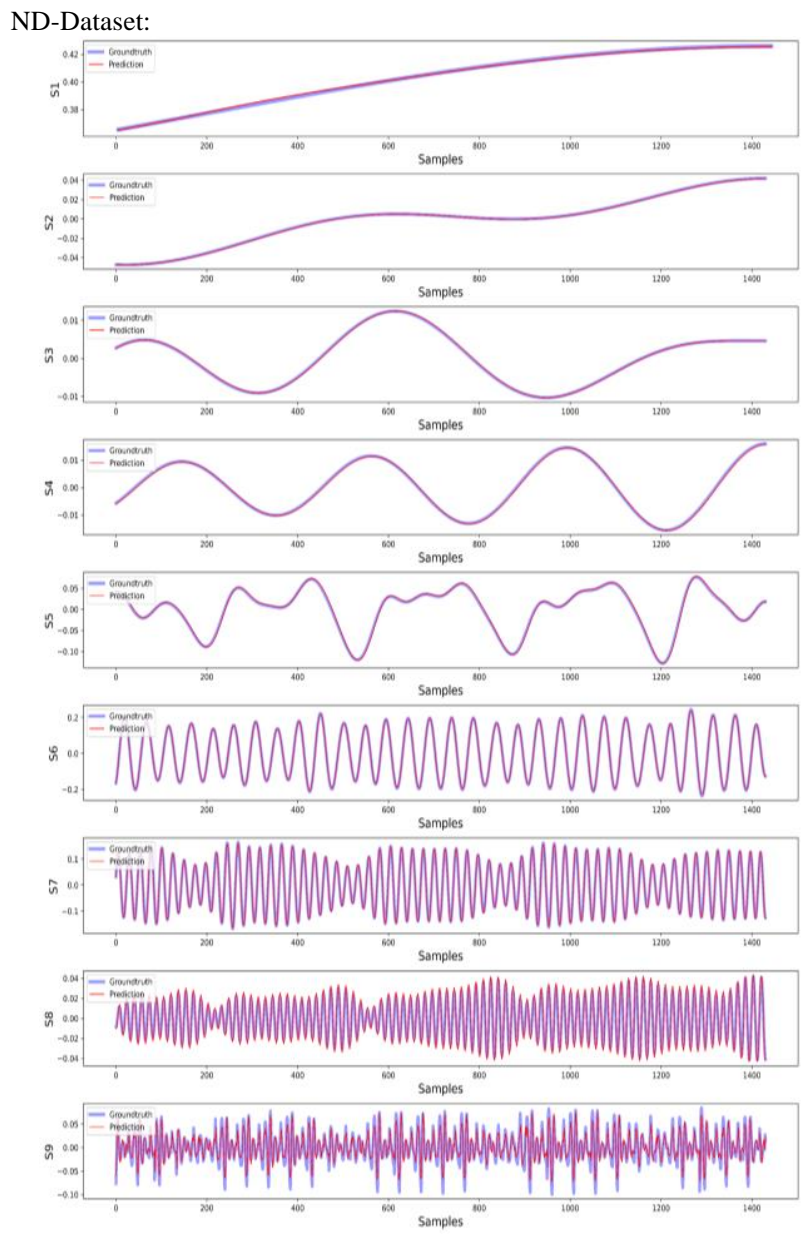

(a)

EWD-Dataset:

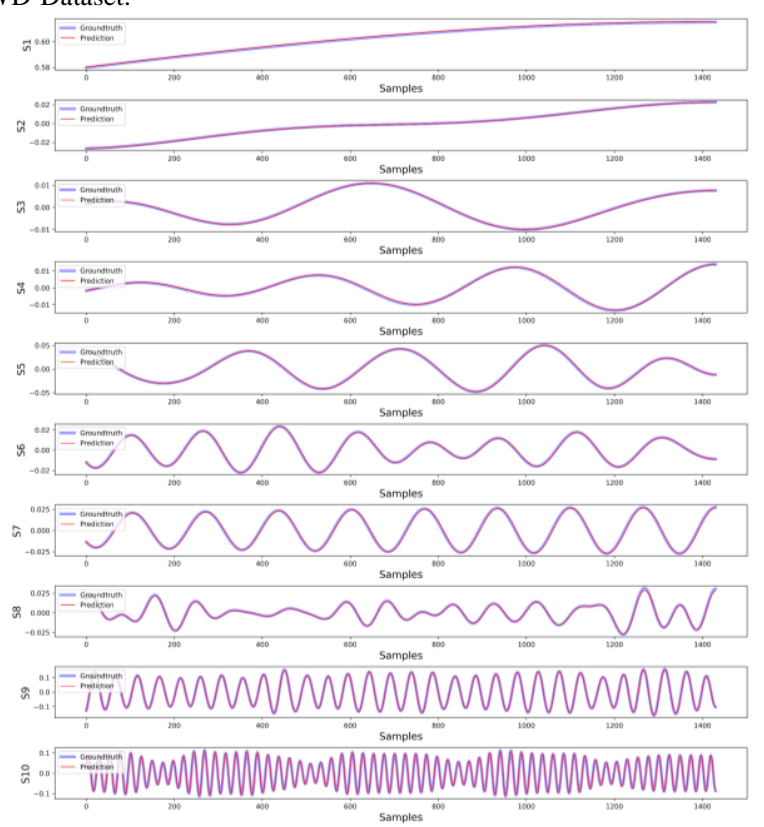




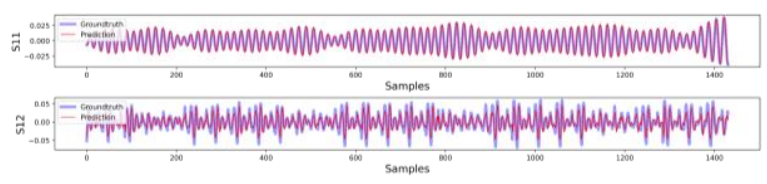

(b)

Fig. 7. Prediction for each sub-layer on validation set (a) ND-dataset with $\mathrm{N}=9$ (b) EWD-dataset with $\mathrm{N}=12$.

\section{Comparison of the Performance of Proposed Method with Other Algorithms}

In this case study, the performance of the proposed NDEWT-BLSTM-BHO is compared with relevant forecasting approaches. Four benchmarks are introduced: 1DCNNBLSTM/BGRU algorithm proposed in [26, 27], VMD-LSTM algorithm proposed in [2, 6, 7], and EMD-LSTM algorithm

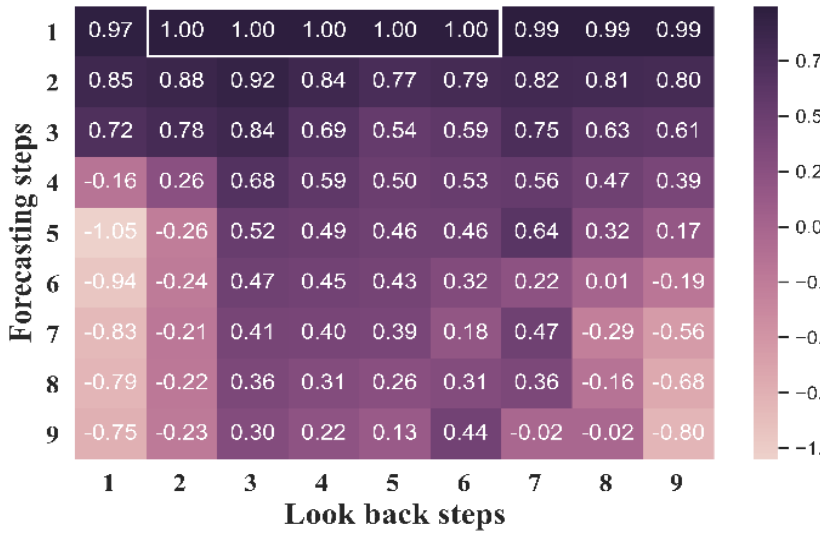

(a)

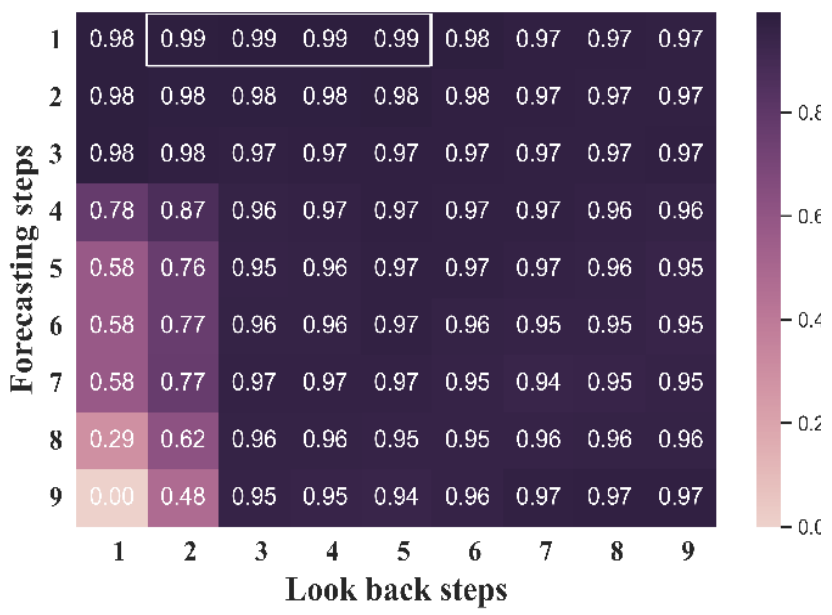

(b)

Fig. 8. $R^{2}$ of the predict performance with different $\mathrm{T}_{\mathrm{L}}$ and $\mathrm{T}_{\mathrm{F}}$ combinations (a) ND-dataset (b) EWD-dataset.

proposed in [2, 18]. A detailed description of models adopted in this study are list as below:

(1) 1D CNN-BLSTM load forecasting model;

(2) $1 \mathrm{D}$ CNN-BGRU load forecasting model;

(3) EMD-LSTM load forecasting model;

(4) VMD-LSTM load forecasting model;

(5) ND-EWT-BLSTM-BOA load forecasting model.

For models (1)-(2), the original time-varying load demand is adopted as the inputs of neural network models, while in the models (3)-(4), the original load demand data is decomposed via EMD/VMD/EWT, then the neural network is training for each sub-layer.

Table 4 and 5 show the performance of five models considering performance metrics (MAE, MAPE, RMSE, $R^{2}$ ) of forecasted load demand given ND-Dataset and EWD-dataset. As shown in the tables, the proposed ND-EWT-BLSTM-BOA outperforms other algorithms, which leads to a smaller value of MAE, MAPE and RMSE, and a larger value of $R^{2}$. For 1DCNN-BLSTM and 1DCNN-BGRU models, the MAPEs of the two datasets are in the range of $1.001 \%-1.031 \%$, and $2.892 \%-3.543 \%$, respectively. While the MAPEs VMD-LSTM and EMD-LSTM are smaller, in the range of $2.533 \%-3.040 \%$, and $3.012 \%-4.260 \%$. The MAPE of the proposed model is the smallest among all models, reaches $0.914 \%$ and $0.558 \%$, showing that the proposed model has better performance than the benchmarks. The same statistical analysis is made for MAE and RMSE values. The proposed model improves RMSE by $66.59 \%$ and $84.06 \%$ for the two datasets comparing with the worst-performing algorithms. For MAEs, the proposed model can improve MAE by $69.62 \%$ and $85.96 \%$ for the two datasets. As for $R^{2}$, the proposed model also achieves the highest values, 0.996 and 0.994 for two datasets.

The results obtained by BLSTM models for all sub-layers are reconstructed to get the forecasting of future load demand. Fig. 9 compares the forecasted values with the testing set by using the proposed model and benchmark models. As can be observed, the results predicted by the proposed model are the closest to the ground truth measurements, in both ND-Dataset (Fig. 9 (a)) and EWD-Dataset (Fig. 9 (b)). Moreover, the results estimated by the EMD-LSTM model are farthest from the ground truth curve, showing that EMD-LSTM performances worst among all algorithms. Fig. 10 shows the scatter plot of the ground truth values and forecasting values of different forecasting models. The scatter plot shows the correlation relationship between two variables, the higher the $R^{2}$ value is the stronger correlation between the predictions and ground truth, representing a better accuracy the forecasting model achieves. As for the proposed model, the scatter about the line is quite small and most points are on the regression line, with only several data values that are far away from other data values. Other models, except for EMD-LSTM, also shows a strong correlation, with $R^{2}$ values over 0.90. And EMD-LSTM shows the worst correlation from the scatter plot, with $R^{2}$ values 0.965 and 0.755 for the two datasets.

TABLE IV

PREDICTION PERFORMANCE OF THE PROPOSED MODEL AND RELATED WORKS (ND-DATASET).

\begin{tabular}{lllll}
\hline \hline Method & MAE (GW) & MAPE (\%) & RMSE (GW) & R2 \\
\hline $\begin{array}{l}\text { 1DCNN- } \\
\text { BLSTM [26, }\end{array}$ & 341.804 & 1.001 & 476.692 & 0.995 \\
$27]$ & & & \\
$\begin{array}{l}\text { 1DCNN-BGRU } \\
{[27]}\end{array}$ & 347.328 & 1.031 & 474.065 & 0.995 \\
$\begin{array}{l}\text { VMD-LSTM } \\
{[2,9,10]}\end{array}$ & 862.053 & 2.533 & 1108.344 & 0.976
\end{tabular}




\begin{tabular}{lllll}
$\begin{array}{l}\text { EMD-LSTM }[2, \\
\text { 5] }\end{array}$ & 871.351 & 3.040 & 1005.477 & 0.965 \\
$\begin{array}{l}\text { Proposed } \\
\text { Method }\end{array}$ & $\mathbf{2 7 1 . 2 4 3}$ & $\mathbf{0 . 9 1 4}$ & $\mathbf{3 7 0 . 3 1 2}$ & $\mathbf{0 . 9 9 6}$ \\
\hline
\end{tabular}

TABLE V

PREDICTION PERFORMANCE OF THE PROPOSED MODEL AND RELATED WORKS (EWD-DATASET)

\begin{tabular}{lllll}
\hline \hline Method & MAE (GW) & MAPE (\%) & RMSE (GW) & R2 \\
\hline $\begin{array}{l}\text { 1DCNN- } \\
\text { BLSTM [26, } \\
\text { 27] }\end{array}$ & 775.498 & 2.892 & 1095.129 & 0.972 \\
$\begin{array}{l}\text { 1DCNN- } \\
\text { BGRU [27] }\end{array}$ & 979.520 & 3.543 & 1709.405 & 0.932 \\
$\begin{array}{l}\text { VMD-LSTM } \\
{[2,9,10]}\end{array}$ & 885.156 & 3.012 & 1140.496 & 0.965 \\
$\begin{array}{l}\text { EMD-LSTM [2, } \\
\text { 5] }\end{array}$ & 1350.518 & 4.260 & 1610.392 & 0.755 \\
$\begin{array}{l}\text { Proposed } \\
\text { Method }\end{array}$ & $\mathbf{1 8 9 . 6 1 4}$ & $\mathbf{0 . 5 5 8}$ & $\mathbf{2 5 6 . 7 0 9}$ & $\mathbf{0 . 9 9 4}$ \\
\hline \hline
\end{tabular}

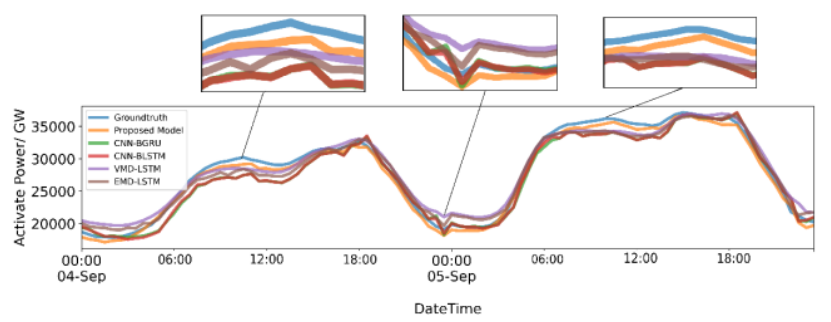

(a)

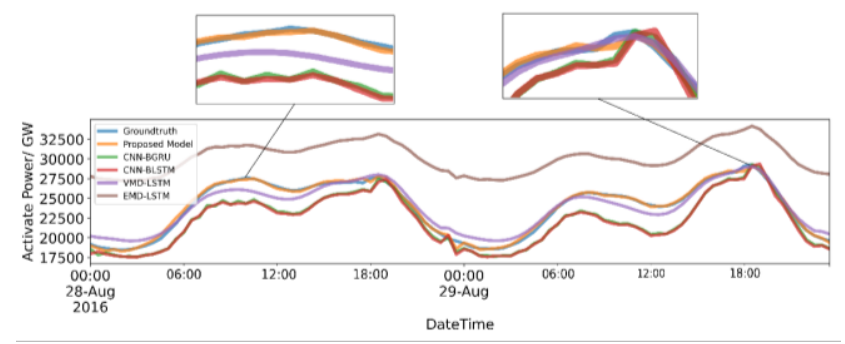

(b)

Fig. 9. Load forecasting performance of the proposed model and other algorithms (a) ND-dataset (b) EWD-dataset.

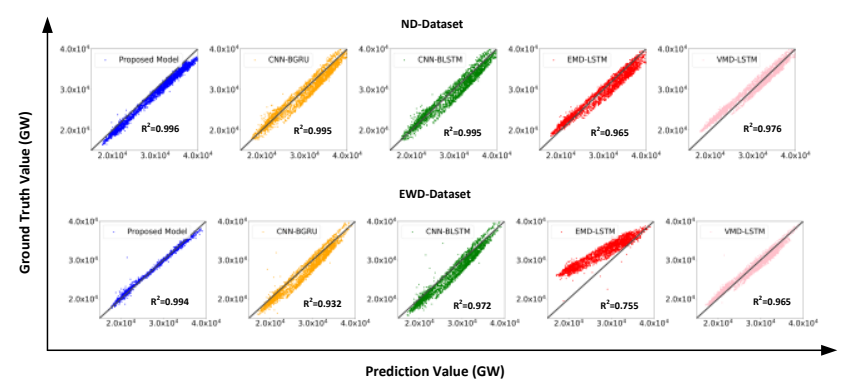

REFERENCE

[1] X. Shi, X. Lei, Q. Huang, S. Huang, K. Ren, and Y. Hu, "Hourly day-ahead wind power prediction using the hybrid model of variational model decomposition and long short-term memory,"
Fig. 10. High-density scatter plot of ground truth values and prediction values of step head load forecasting models.

\section{CONCLUSIONS}

In this paper, a hybrid load forecasting model (ND-EWTBLSTM-BHO) is proposed, the model considers wavelet-based denoising algorithm, EWT component decomposition technique, bidirectional LSTM algorithm, and Bayesian hyperparameter optimization algorithm. The proposed model first filters noises such as electric spikes from the measured load demand data. Then an EWT algorithm is adopted to decompose the data into $N$ sub-layers to extract time and frequency domain features. $N$ BLSTM neural network models are trained for all sub-layers as the next step. Moreover, a BHO algorithm is used for tuning the hyperparameters to find the best combinations of hyperparameters that achieve the best performance. Finally, the prediction results for all sub-layers are reconstructed and present the result of the load forecasting. The British load demand dataset is used for the simulation, both the national demand (ND-dataset) and the demand of England and Wales areas (EWD-Dataset) are fully investigated. In this paper, three case studies are demonstrated. The first case study aims to optimize sub-layer number $N$ to achieve the best performance. Referring to the simulation, it is observed that when $N$ ranges between 9 and 12, the proposed load forecasting model achieve the smallest RMSE, MAPE, and MAE values. As for the second case study, the impacts of look-back step $T_{L}$ as well as the forecasting steps $T_{F}$ are studied. From the results presented in Fig. 7, a conclusion is obtained that the proposed model has a good forecasting ability even to forecasting load demand nine hours later. when $T_{L}$ ranges $2-6$, and $T_{F}$ ranges 1-2, the model achieves the highest accuracy. In the last case study, the optimized model is compared with other forecasting models (1DCNN-BLSTM, 1DCNN-BGRU, VMD-LSTM, EMDLSTM). From the results, it is shown that the proposed model improves RMSE by $66.59 \%$ and $84.06 \%$, improves MAE by $69.62 \%$ and $85.96 \%$, and improves MAPE by $69.93 \%$ and $86.90 \%$ for the two different datasets, respectively. The conclusion is made that the proposed model has better performance than existing component decomposition models. In the future, more variables that influence the load demand will be added to the forecasting model (meteorological data, geographic data, etc.) to improve the flexibility of the proposed model. In addition, the integration of renewable energy will be taken into consideration since the distributed generation will bring more uncertainty to the load forecasting.
Energies, vol. 11, no. 11, p. 3227, 2018.

[2] S. Kim, G. Lee, G.-Y. Kwon, D.-I. Kim, and y.-j. Shin, "Deep Learning Based on Multi-Decomposition for Short-Term Load Forecasting," Energies, vol. 11, p. 3433, 12/07 2018.

[3] R. J. Hyndman and G. Athanasopoulos, Forecasting: principles and practice. OTexts, 2018.

[4] H. Liu and Z. Long, "An improved deep learning model for predicting stock market price time series," Digital Signal 
Processing, vol. 102, p. 102741, 2020

Y. K. Semero, J. Zhang, and D. Zheng, "EMD-PSO-ANFIS-based hybrid approach for short-term load forecasting in microgrids," IET Generation, Transmission Distribution, vol. 14, no. 3, pp. 470475, 2019.

[6] Q. Liu, Y. Shen, L. Wu, J. Li, L. Zhuang, and S. Wang, "A hybrid FCW-EMD and KF-BA-SVM based model for short-term load forecasting," CSEE Journal of Power Energy Systems, vol. 4, no. 2, pp. 226-237, 2018.

[7] Z. Zhu, Y. Sun, and H. Li, "Hybrid of EMD and SVMs for shortterm load forecasting," in 2007 IEEE International Conference on Control and Automation, 2007, pp. 1044-1047: IEEE.

[8] W. Li, C. Quan, X. Wang, and S. Zhang, "Short-Term Power Load Forecasting Based on a Combination of VMD and ELM," Polish Journal of Environmental Studies, vol. 27, no. 5, 2018.

[9] G. Zhang et al., "Load Prediction Based on Hybrid Model of VMD-mRMR-BPNN-LSSVM," Complexity, vol. 2020, 2020.

[10] F. He, J. Zhou, Z.-k. Feng, G. Liu, and Y. Yang, "A hybrid shortterm load forecasting model based on variational mode decomposition and long short-term memory networks considering relevant factors with Bayesian optimization algorithm," Applied energy, vol. 237, pp. 103-116, 2019.

[11] F. He, J. Zhou, L. Mo, K. Feng, G. Liu, and Z. He, "Day-ahead short-term load probability density forecasting method with a decomposition-based quantile regression forest," Applied Energy, vol. 262, p. 114396, 2020.

[12] J.-p. Liu and C.-1. Li, "The short-term power load forecasting based on sperm whale algorithm and wavelet least square support vector machine with DWT-IR for feature selection," Sustainability, vol. 9 , no. 7 , p. 1188,2017

[13] J. Gilles, "Empirical wavelet transform," IEEE transactions on signal processing, vol. 61, no. 16, pp. 3999-4010, 2013.

[14] T. Zhao and J. Li, "Application of empirical wavelet transform in power load forecasting," Proc. Chinese Automation Congr, pp. 4009-4013, 2018.

[15] W. Liu and W. Chen, "Recent advancements in empirical wavelet transform and its applications," IEEE Access, vol. 7, pp. 103770103780, 2019.

[16] K. Thirumala, A. C. Umarikar, and T. Jain, "Estimation of singlephase and three-phase power-quality indices using empirical wavelet transform," IEEE Transactions on power delivery, vol. 30, no. 1, pp. 445-454, 2014.

[17] G. Luo, D. Zhang, and D. Baleanu, "Wavelet denoising," Advances in wavelet theory their applications in engineering, physics technology, p. 634, 2012.

[18] S. Hochreiter and J. J. N. c. Schmidhuber, "Long short-term memory," vol. 9, no. 8, pp. 1735-1780, 1997.

[19] O. Kramer, D. E. Ciaurri, and S. Koziel, "Derivative-Free Optimization," in Computational Optimization, Methods and Algorithms, S. Koziel and X.-S. Yang, Eds. Berlin, Heidelberg: Springer Berlin Heidelberg, 2011, pp. 61-83.

[20] A. H. Victoria and G. Maragatham, "Automatic tuning of hyperparameters using Bayesian optimization," Evolving Systems, 2020/05/25 2020.

[21] "Historic Demand Data," N. Grid, Ed., ed, 2020.

[22] G. R. Lee, R. Gommers, F. Waselewski, K. Wohlfahrt, and A. O'Leary, "PyWavelets: A Python package for wavelet analysis," Journal of Open Source Software, vol. 4, no. 36, p. 1237, 2019.

[23] D. Laszuk, "PyEMD Documentation," ed, 2020.

[24] V. R. Carvalho, M. F. Moraes, A. P. Braga, and E. M. Mendes, "Evaluating five different adaptive decomposition methods for EEG signal seizure detection and classification," Biomedical Signal Processing Control, vol. 62, p. 102073, 2020.

[25] J. Bergstra, D. Yamins, and D. D. Cox, "Hyperopt: A python library for optimizing the hyperparameters of machine learning algorithms," in Proceedings of the 12th Python in science conference, 2013, vol. 13, p. 20: Citeseer.

[26] W. Kong, Z. Y. Dong, Y. Jia, D. J. Hill, Y. Xu, and Y. Zhang, "Short-term residential load forecasting based on LSTM recurrent neural network," IEEE Transactions on Smart Grid, vol. 10, no. 1, pp. 841-851, 2017.

[27] M. Sajjad et al., "A novel CNN-GRU-based hybrid approach for short-term residential load forecasting," IEEE Access, vol. 8, pp. 143759-143768, 2020. 